\title{
Just another case of neck pain? A case report of acute retropharyngeal calcific tendinitis
}

\author{
Emily Le Souder, BSc ${ }^{*}$; Michael M. Beyea, MD, $\mathrm{PhD}^{\dagger \neq}$; Robert J. Sedran, MD, MSc, BESc ${ }^{\dagger}$
}

Keywords: Case report, neck pain, retropharyngeal tendinitis

breathing, or any focal neurologic symptoms, including weakness or paresthesia in the extremities. She denied any trauma, recent travel, or sick contacts. Her past medical history was unremarkable, and she was not taking

\section{INTRODUCTION}

Neck pain is a common presentation to the emergency department (ED), and its etiology can range from benign to acutely life-threatening.

Acute retropharyngeal calcific tendinitis is a rare ED diagnosis but may be more common than originally thought. ${ }^{1}$ It is caused by the deposition of calcium hydroxyapatite in the Longus colli tendon in the neck. ${ }^{2,3}$

We present a case of a patient who initially presented to the ED with nonspecific neck pain but was found to have acute retropharyngeal calcific tendinitis. This case demonstrates the nonspecific presentation of this condition and the need to consider it in order to avoid over- or under-treating patients presenting to the ED with neck pain.

\section{CASE REPORT}

A healthy 24-year-old female presented to the ED with a 2-day history of constant worsening neck pain. The pain was significantly exacerbated by neck movement. She also noted odynophagia but denied throat pain or anterior neck pain. When asked to localize the pain when swallowing, she pointed to her lateral neck, over her trapezius muscles bilaterally. Initially, she refused to drink in the ED due to pain.

She denied any fever, chills, night sweats, and malaise. She denied any shortness of breath, increased work of any prescription medications. She denied any smoking, alcohol, and other substance use.

Her triage vitals were stable, and she was afebrile. On examination, she appeared well and was in no distress; however, she protected her neck in a stiff and neutral position. A musculoskeletal exam revealed decreased flexion, extension, and rotation of the neck due to pain. She was tender to palpation over the trapezius muscles bilaterally. There was no spinal tenderness in the cervical or thoracic regions. There were no palpable neck masses or lymphadenopathy. Examination of the oropharynx was benign. The neurologic exam was unremarkable.

Initially, it was thought that her pain was musculoskeletal in origin and was likely related to muscle tension; however, the description of her pain being exacerbated with swallowing was not in keeping with this diagnosis prompting further investigation.

Laboratory testing revealed a minimally elevated C-reactive protein (CRP) of 7.1 and a white blood cell count of 11.5 but was otherwise non-contributory. A neck soft-tissue X-ray showed thickening of the prevertebral soft tissues at the upper cervical level (Figure 1, A). A computed tomography (CT) scan revealed a small linear fluid collection in the retropharyngeal space extending through $\mathrm{C} 1$ to $\mathrm{C}$, which was not suggestive of abscess formation (Figure 1, B). A notation was made of a tiny calcific spur at the anterior arch of $\mathrm{C} 1$ inferiorly. The trapezius muscle and the rest of the neck musculature appeared unremarkable. These findings were in keeping with acute retropharyngeal calcific tendinitis.

From the *Schulich School of Medicine and Dentistry, Western University, London, ON; †Division of Emergency Medicine, London Health Sciences Centre, Western University, London, ON; and the $¥$ Critical Care Medicine, London Health Sciences Centre, Western University, London, ON..

Correspondence to: Dr. Robert Sedran, Emergency Medicine, Victoria Hospital, LHSC Rm E1-102, 800 Commissioners Rd. E, London, ON N6A 5W9; Email: rsedran@uwo.ca

(c) Canadian Association of Emergency Physicians

CJEM 2019;21(4):554-556

DOI 10.1017/cem.2019.9 


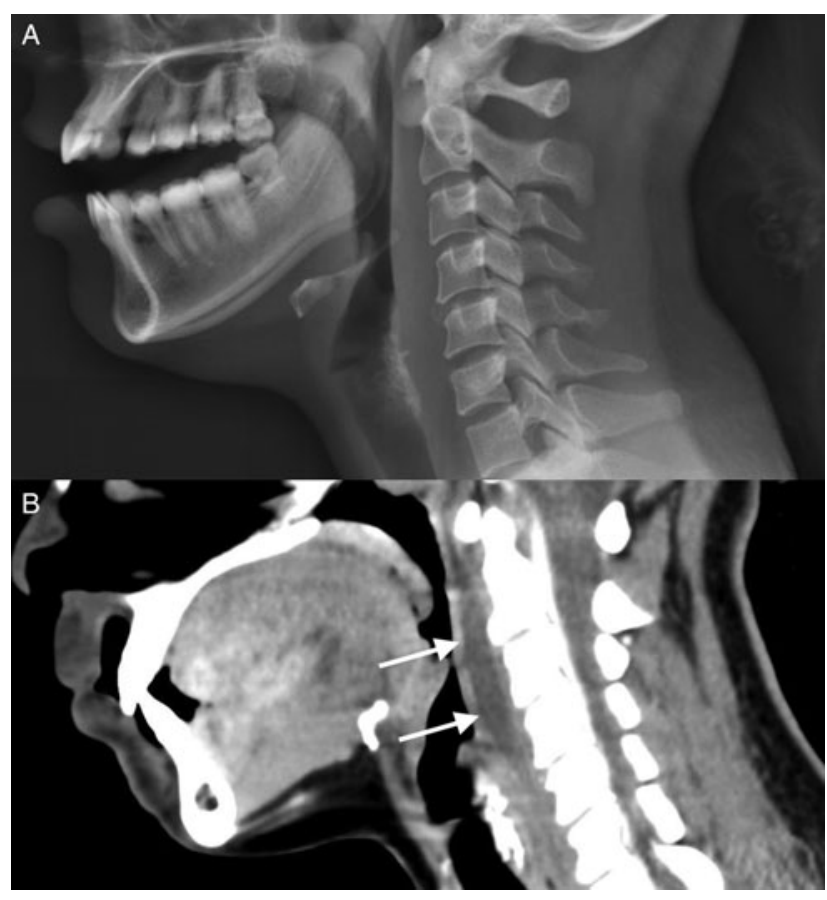

Figure 1. Neck soft-tissue $X$-ray showing thickening of the pre-vertebral soft tissues at the upper cervical level (A) and a CT scan revealing linear fluid in the retropharyngeal space extending through $\mathrm{C} 1$ to $\mathrm{C} 4(\mathrm{~B})$.

The patient was treated with ibuprofen in the ED and her pain was sufficiently reduced to tolerate oral fluids at the time of discharge. She was discharged with a prescription for naproxen and a 10-day course of amoxicillin/clavulanic acid, $500 \mathrm{mg}$ TID, which was empirically chosen after reviewing the case with the otolaryngologist consultant on call. The patient was asked to return if her symptoms did not improve within 48 hours or if she developed a respiratory compromise. She had no subsequent presentations to the hospital.

\section{DISCUSSION}

Acute retropharyngeal calcific tendinitis is a rare diagnosis in the ED; however, recent literature suggests that this condition may be common but underdiagnosed due to its nonspecific presentation and inconsistency in patients presenting for medical care. ${ }^{1}$ The purpose of this case report is to disseminate knowledge of acute retropharyngeal calcific tendinitis as a differential diagnosis of nonspecific neck pain and odynophagia. This may help avoid over- or under-treating conditions, such as retropharyngeal abscesses and meningitis, which can present with neck pain. Consideration of this diagnosis could also aid in decreasing patient anxiety and managing patient expectations.

Acute retropharyngeal calcific tendinitis is caused by the deposition of calcium hydroxyapatite crystals in the Longus colli muscle, which is located in the prevertebral space extending from the atlas to the third thoracic vertebra and contributes to neck flexion. ${ }^{2,3}$ This phenomenon was first described in 1964 by Hartley et al. ${ }^{4}$ The calcium deposition contributes to an inflammatory response and an acute tendonitis. The etiology of this deposition is poorly understood but may be due to repetitive neck trauma. ${ }^{5}$

Decreased neck range of motion, neck pain, neck stiffness, odynophagia, dysphagia, and throat swelling have been described as presenting symptoms. ${ }^{1,2,4}$ Low grade fever, mild leukocytosis, and elevated CRP may also be found. ${ }^{1,2,5}$ An observational study of retropharyngeal calcific tendonitis by Horowitz et al., including 13 patients, quoted incidence of this condition at 0.5 cases per 100,000-person years. ${ }^{1}$

CT is important to differentiate this diagnosis from a retropharyngeal abscess. Suggestive CT findings include a calcific pre-vertebral density at the $\mathrm{C} 1-\mathrm{C} 2$ level and associated soft tissue swelling, which can extend to C6. ${ }^{2,3}$ Pre-vertebral swelling may be seen on a lateral neck $\mathrm{X}$-ray but does not provide a definitive diagnosis., $2,3,5$

Acute retropharyngeal calcific tendinitis has been described as a self-limiting condition that resolves spontaneously within 2 weeks; however, the pain can be quite debilitating. ${ }^{1,3,5}$ If symptoms persist or are accompanied by other symptoms, such as meningismus or high fever, an alternative diagnosis should be considered. A short course of nonsteroidal anti-inflammatories and avoidance of aggravating neck movements are recommended. ${ }^{2,5}$ A short course of oral steroids may be considered in severe cases. ${ }^{5}$ There is no evidence to support the use of antibiotics, and they are likely not indicated in this inflammatory condition. ${ }^{1}$ However, due to a lack of familiarity with this condition and possible alternative diagnoses, such as a retropharyngeal abscess, antibiotics have been given empirically as a precaution, in some cases. ${ }^{1}$

\section{CONCLUSION}

Acute retropharyngeal calcific tendinitis is rarely diagnosed in the ED but may be a common etiology of neck pain and odynophagia. Considering this diagnosis 
could help manage patient expectations and prevent overtreatment or under treatment of patients presenting with neck pain.

Competing interests: None declared.

\section{REFERENCES}

1. Horowitz G, Ben-Ari O, Brenner A, et al. Incidence of retropharyngeal calcific tendinitis (Longus colli tendinitis) in the general population. Otolaryngol Head Neck Surg 2013;148(6):955-8.
2. Coulier B, Macsim M, Desgain O. Retropharyngeal calcific tendinitis-Longus colli tendinitis-an unusual cause of acute dysphagia. Emerg Radiol 2011;18(5):449-51.

3. Pollak N, Wexler S. Retropharyngeal calcific tendonitis mimics a retropharyngeal abscess. Case Rep Otolaryngol 2013; epub, 1-4.

4. Hartley J. Acute cervical pain associated with retropharyngeal calcium deposit. J Bone Joint Surg 1964;46(8):1753-4.

5. Zapolsky N, Heller M, Felberbaum M, et al. Calcific tendonitis of the Longus colli: An uncommon but benign cause of throat pain that closely mimics retropharyngeal abscess. 7 Emerg Med 2017;52(3):358-60. 